Many of us would be saddened if we really have already hit upon giant squid as big, or almost as big, as they get. Not that an animal proven to reach a length of 15 or 20 feet, with tentacles extending to 50 , is by any means negligible; it is just that, deep down, we would like to believe that there are creatures the size of submarines waiting to grab at us in the unexplored wilderness of the deep sea.

So Ellis sets out to keep us all happy, by quoting sailors' tales, many - but let's hope not all of them - gross exaggerations, as well as taking a hard-headed look at the evidence of the specimens washed up on beaches, trawled from the depths or recovered from the stomachs of sperm whales. Sperm whales are a lot better at catching these creatures than we are ("we only catch the slow, the sick, and the stupid" - again a quote, this time from Clyde Roper, at the Smithsonian Institution in Washington DC). Fortunately, sperm whales have difficulty digesting the beaks of squid, so we know something of the sizes, distribution and probable numbers of Architeuthis from their gut contents - a source of information, incidentally, that has dried up with the moratorium on whaling.

It seems that giant squid (Architeuthis is not the only genus of big squid, although it seems to include all the real monsters) are very widely distributed, and probably quite common. Sperm whales seem to eat an awful lot of them and, at least when adult, the big squid prefer cold, deep waters. So the ones we find at the surface are perhaps always moribund.

The sad fact emerges that nobody has ever seen a giant squid in good condition. And most of the available evidence suggests that the animals are probably relatively slow moving, drifters rather than drivers. They are neutrally buoyant, because much of their muscle tissue is replaced by cells retaining ammonium chloride - which is lighter than sea water and, incidentally, makes them thoroughly unpalatable to us. The two tentacles responsible for most of the total length of the giants are probably far too long and thin to be shot out at prey in the manner of the cuttlefish and smaller squid that we observe in aquaria. There is a surprising lack of the giant nerve fibres that would ensure that the mantle contracts simultaneously all over, and the cartilage locking the mantle to the funnel is poorly developed, so that a really powerful jet is perhaps beyond them.

Ellis's book tells us all this, as well as listing all the authenticated sightings and strandings of Architeuthis, and reviewing the history of the many sea-serpents that were probably, in fact, squid. There are chapters on the giant squid in literature and in films and about the several reconstructions of giant squid made for museums throughout the world - Hitler, he reminds us, destroyed the model in the Natural History Museum in London.

There are plenty of illustrations from imaginative accounts of contacts in the past and a liberal sprinkling of photographs of men in white coats standing around (and usually somewhat behind - even scientists in white coats really want squids to be big) the laid-out remains of more recent encounters. A gold-mine of fact and fantasy, for we scientists who work on cephalopods and for all of us who love monsters.

Martin Wells is in the Department of Zoology, University of Cambridge, Cambridge CB2 1TN, UK.

\section{The cosmic rain}

\section{The Particle Century
edited by Gordon Fraser}

Institute of Physics Publishing: 1998. 227 pp. $\$ 59.50, £ 30$

\section{David Miller}

Among the authors of these 20 essays there are sufficient 'good communicators' that the serious reader can learn a great deal about the science, and there are sufficient 'great names' to give a thrill of authenticity from the first-hand accounts of discoveries. And some of the 'great names' are among the best communicators. The story really starts with two theoretical insights, Paul Dirac's prediction of antielectrons and the assertion by Hideki Yukawa that the short-range force that binds protons and neutrons into nuclei must begenerated by the exchange of a massive particle.

Both were confirmed by observations of cosmic ray interactions: the positron (the anti-electron) very soon after its prediction; the pion (Yukawa's force carrier) only after a long delay from 1935 to 1947 , despite the early discovery of a 'mesotron' with many but not all - of the right properties. This later turned out to have been the muon the first heavy copy of the electron, as explained in the essay on the "Cosmic Rain" by Owen Lock, who began his PhD work at Bristol just after Cecil Powell had identified the pion as a separate object from the muon.

Cosmic rays also generated the first handfuls of strange particles, but they were only properly understood after accelerators generated hundreds of them, and bubble chambers pictured their behaviour. Nick Samios writes about the quest to observe the whole set of strange particles which culminated in his team's discovery in 1964 of the omega-minus, whose properties had already been predicted from SU(3) theory. One of those who made the prediction was Yuval Ne'eman, who tells how falteringly the idea of quarks grew from the mathematical structure of SU(3) into a physical hypothesis. Physicists only began to believe that the three quarks of SU(3) (up, down and strange) were real after seeing deep inelastic scattering of electrons and neutrinos from pointlike objects inside protons described here by Jerry Friedman and Henry Kendall, two leaders of the team who did the first such experiment.
That was the beginning of the revolution of the early 1970s, when the whole structure of the present Standard Model began to fall into place, driven by a rapid dialogue between experimental surprises and theoretical hypotheses. Roy Schwitters describes how the discovery of a spin 1 resonance, the $\psi / \mathrm{J}$, was matched to a theory of weak interactions which needed a fourth (charm) quark which could bind to its own antiquark to make just such a spin 1 state. Once charm was understood it became possible to unify the weak and electromagnetic theories into a single gauge-field theory - described in a superbly lucid essay by Martinus Veltman, one of its founders. This 'electroweak' theory needed the $\mathrm{W}$ and the $\mathrm{Z}$ bosons as its force carriers. They are about 100 times heavier than a proton - which makes them very hard to produce. Carlo Rubbia explains in his quirky English how he and colleagues built a machine to collide protons and antiprotons at sufficiently high energy to prove that the $\mathrm{W}$ and $\mathrm{Z}$ existed. At the same time a gauge-field theory of the strong interaction between quarks developed, Quantum Chromodynamics (QCD), discussed by Sau Lan Wu, one of the team that proved the existence of the gluon, the gauge-boson which carries the strong force.

There are healthy overlaps between authors. If a reader does not understand a concept on first encounter - say the need for a top quark in Guido Altarelli's essay on how the Standard Model works - it might become clearer after reading Mel Shochet. Some essays are straight story-telling, some round out the picture by explaining the theory, or how the accelerators and detectors work. Even at the end of the century there are gaps in the Standard Model: is there a Higgs boson, how do we fit gravity in, why are some symmetries almost but not quite exact? As John Ellis points out, these gaps link up with profound questions in cosmology.

The first essay is different from all the rest, and extremely valuable. Catherine Westfall and John Krige give a brief social and political history of post-war physics. They show, among other things, how in the United States the rivalries of the Cold War drove science along, while in Europe science was a neutral ground on which enmities could be forgotten, leading to the still growing success of the European Laboratory for Particle Physics (CERN) in Switzerland. And there are warnings about how governments now want to 'acquire' science rather than to work in partnership with the scientist. This book could do a little to help restore that partnership. The dedicated non-scientist will get a lot from it, although its main audience is likely to be among school and undergraduate students of physics. David Miller is in the Department of Physics and Astronomy, University College London, Gower Street, London WC1E 6BT, UK. 